\title{
Quadratic Greedy Algorithm for Multichannel Access in Cognitive Radio Network
}

\author{
Soumaya Dahi, Sami Tabbane \\ Mediatron, Higher School of Communications of Tunisia (Sup'Com) \\ Parc Technologique des Communications de Ghazala \\ 2083 Tunis - Tunisia
}

\begin{abstract}
Spectrum handoff is one of the main issues in cognitive radio networks. Spectrum handoff occurs when the owner of the spectrum or the primary user reclaims a channel already used opportunistically by a secondary user. Such handoff becomes more frequent especially in case of multichannel access since that in a wider band, a PU is likely to reclaim a part of the band more quickly. This process has a negative impact on the performance of SU transmission in terms of delay and link maintenance. In this paper, the problem of minimizing the secondary user handoff process because of primary user activity is underlined. An analytical formulation based on knapsack problem is established and a new spectrum selection scheme is proposed. The main idea is to maximize jointly the holding time and the achievable throughput of the selected channels. The performance of the proposed access model is investigated through numerical simulations.
\end{abstract}

\section{Keywords}

Handoff, Holding Time, Rate, Knapsack problem, heuristic, quadratic greedy algorithm.

\section{CONTEXT/MOTIVATIONS}

Cognitive radio (CR) technology is regarded as a promising solution to the spectrum scarcity problem. The key idea of this paradigm is to allow unlicensed Secondary Users (SUs) to utilize opportunistically "spectrum holes" with the main condition to not interfere with the licensed Primary User (PU) transmission.

However, due to the spectrum varying nature of CR networks, unlicensed users are required to perform spectrum handoff (HO), which refers to the process that when the current channel used by an SU is no longer available, the SU needs to pause its on-going transmission, vacate that channel, and determine a new available channel to continue the transmission.

Clearly, many interruptions from the primary users specially in case of multichannel access will result in multiple handoffs, thereby affects negatively the performance of the SU transmission in term of delay and link maintenance. In fact, in normal conditions of licensed usage, channel switching can cause a packet loss ratio of 3\% [1], this ratio becomes worse in CR usage because of the unpredictable behavior of PU. Besides, handoff generates additional delay since for each frequency switching a sensing phase has to take place, additionally to channel evacuation and link set up.

Although reducing the $\mathrm{HO}$ due to $\mathrm{PU}$ activity is crucial for secondary transmission, limited studies consider this issue compared with other functionalities (spectrum sensing, spectrum management, and spectrum sharing) [2] of CR networks.

Moreover, in the literature, most of research works regarding spectrum handoff focused on the physical-layer impacts without considering the effect in the MAC layer. Most of MAC protocol design for spectrum handoff falls into two categories based on the moment when SUs carry out spectrum handoffs. In the first category, SUs perform channel switching after detecting the reappearances of PUs, namely the reactive approach [3][4][5]. In the other category, SUs predict the future PU channel activities based on observed channel usage statistics and perform spectrum handoffs before the disruptions with PU transmissions, namely the proactive approach [6][7].

However, either for reactive or proactive $\mathrm{HO}$ process when SUs perform spectrum handoffs a well-designed channel selection method is required to avoid the disruption of $\mathrm{SU}$ transmission because of PU activity.

According to our analysis, it will be interesting to select channels having longer idle periods( Holding Time) but also it will be interesting to transmit on a channel having better quality since that better is the quality, higher is the throughput and less will be the requested holding time to achieve SU transmission.

In this work we propose an analytical formulation of spectrum selection strategy in case of multichannel access, considering this tradeoff between the affordable holding time and the achievable throughput related to the selected channels.

The rest of this paper is organized as follows: in section II, the optimal channels selection problem is formulated mathematically. Section III introduces the proposed heuristic approach to solve this problem. The proposed model is evaluated in Section IV. Finally, Section V gives concluding remarks.

\section{PROBLEM FORMULATION AND ANALYSIS}

\subsection{Problem formulation}

As mentioned before, the aim of this work is to propose a handoff reducing access model for CR networks in case of multichannel access. Then to formalize the problem we start from an approximation of $\mathrm{NHO}(\mathrm{C})$ : the estimated number of $\mathrm{HO}$ to achieve SU transmission when transmitting simultaneously over $\mathrm{C}$ channels. In [8], it has been proven that for large SU transmission time $\operatorname{Tx}(\mathrm{C})$ over $\mathrm{C}$ channels:

$$
\mathrm{NHO}^{(\mathrm{C})}=1+\frac{\mathrm{Tx}^{(\mathrm{C})}}{\mathrm{HT}^{(\mathrm{C})}}
$$

Where $\mathrm{HT}^{(\mathrm{C})}$ is the minimum residual idle time of all $\mathrm{C}$ channels. That is $\mathrm{HT}(\mathrm{C})=\min \{\mathrm{HTi}\} \mathrm{i}=1$..C. 
The accuracy of this approximation has been validated under several common used distributions of idle times [8].

when considering that:

$$
T x^{(C)}=\frac{A_{0}}{\sum_{i=1}^{C} R^{(i)}}
$$

Where

$A_{0}$ is the total amount of data the SU wants to transmit.

- $\quad R^{(i)}$ is the SU's achievable throughput over channel i.

Then

$$
N H O^{(C)}=1+\frac{A_{0}}{\sum_{i=1}^{C} R^{(i)} \times H T^{(C)}}
$$

Then to minimize $\mathrm{NHO}^{(\mathrm{C})}$, it is necessary to maximize $\sum_{\mathrm{i}=1}^{\mathrm{C}} \mathrm{R}^{(\mathrm{i})} \times \mathrm{HT}^{(\mathrm{C})}$ which corresponds to the achievable amount of data during $\mathrm{HT}^{(\mathrm{C})}$. Then $\mathrm{SU}$ is interested on selecting channels having highest HT but also highest achievable rate. This allows transmitting data in less time during longer periods.

However, for the sake of efficient usage of spectrum holes, it would be interesting to penalize channels whose (HT, R) is much larger than effectively needed for SU transmission. This constraint may be expressed as follows:

$$
\sum_{i=1}^{C} R^{(i)} \times H T^{(C)} \leq \alpha \times A_{0}
$$

Where $\alpha$ is an adjustable parameter absorbing the estimation error of $(\mathrm{HT}, \mathrm{R})$.

Thus, if we consider an access model where the SU has to choose $\mathrm{C}$ channels among $\mathrm{N}$ to transmit on, the optimal channel set $\mathrm{X}^{*}$ selection problem is expressed as follows:

$$
\begin{aligned}
& X^{*}=\operatorname{argmax} \sum_{i=1}^{N} x_{i} R^{(i)} \times \min \left\{x_{i} H T^{(i)}\right\}_{\substack{i=1 . . N \\
x_{i}=1}} \\
& \text { s.t. }\left\{\begin{array}{c}
\sum_{i=1}^{N} x_{i} R^{(i)} \times \min \left\{x_{i} H T^{(i)}\right\}_{\substack{i=1 . . N \\
x_{i}=1}} \leq \alpha \times A_{0} \\
\sum_{i=1}^{N} x_{i}=C \\
x_{i} \in\{0,1\}
\end{array}\right.
\end{aligned}
$$

The constraint (7) is added to insure that a SU can transmit over exactly $\mathrm{C}$ channels at a given time.

\subsection{Problem Analysis}

The problem formulation above led to a special case of knapsack problem optimization: subset sum problem.

The classical Knapsack Problem (KP) is defined by a set $\mathrm{N}=\{1, \ldots, \mathrm{n}\}$ of items, each having a positive profit $\mathrm{pj}$, and a positive weight wj and by a positive knapsack capacity $\mathrm{K}$. The problem calls for selecting the set of items with maximum overall profit among those whose overall weight does not exceed the knapsack capacity [9]. KP has the following formulation:

$$
\text { Maximize } \sum_{j \in N} p_{j} x_{j}
$$

$$
\text { s.t. }\left\{\begin{array}{c}
\sum_{j \in N} w_{j} x_{j} \leq K \\
x_{j} \in\{0,1\}, j \in N
\end{array}\right.
$$

Where each binary variable $x \mathrm{j}, \mathrm{j} \in \mathrm{N}$,is equal to 1 if and only if item $\mathrm{j}$ is selected.

The Subset Sum Problem (SSP) is the special case of KP arising when $p_{j}=w_{j}$ for each $j \in N$ [10]. In our case items correspond to channels, the capacity $\mathrm{K}$ corresponds to $\alpha \times$ $\mathrm{A}_{0}$ and the weight wj corresponds to $\sum_{\mathrm{i}=1}^{\mathrm{N}} \mathrm{R}^{(\mathrm{i})} \times$ $\min \left\{\mathrm{x}_{\mathrm{i}} \mathrm{HT}^{(\mathrm{i})}\right\}_{\substack{\mathrm{i}=1 . . \mathrm{N} \\ \mathrm{x}_{\mathrm{i}}=1}}$.

SSP can obviously be solved (either exactly or heuristically) by any of the methods deployed for the 0-1 knapsack problem. Namely, the branch and- bound method [11], and dynamic programming [10] [11] can be used to find the exact optimal solution of the problem.

However subset sum, problem deserves specific treatment since all upper bounds generally used for branch and bound algorithm give, for SSP, the trivial value $\mathrm{K}$ (since $\mathrm{pj} / \mathrm{wj}=1$ for all $\mathrm{j}$ ). As a consequence, one would even expect catastrophic behavior of the branch-and-bound algorithms for the 0-1 knapsack problem, degenerating, for SSP, into complete enumeration of possible solutions.

Besides, in our case, we are dealing with a specific optimization problem combining several variants of SSP:

- Oregon Trail Knapsack: defining the value of an item i by a function that is not necessarily a constant, but depending on the presence or absence of another item $\mathrm{j}$ in the knapsack [12]. In our case this function is $\min \left\{x_{i} H T^{(i)}\right\}_{i=1 . . N}$. Then, this inter-item value dependency is an additional complication, since the value of an item may not be known until the end of the search path.

- The Exact k-item SSP (E-kSSP) ) is the variant of SSP where the number of items in a feasible solution must be exactly equal to $\mathrm{k}$ [13]. This is expressed by the cardinality constraint in equation (7).

\section{OPTIMAL CHANNEL SELECTION PROBLEM RESOLUTION}

To the best of our knowledge, no previous work dealt with a so complicated variant of KP problem. Even if an exact 
solution exists, finding it would necessitate either an exhaustive search or a high computation load [9]. That is why it is interesting in this work to consider a heuristic method to approximate the solution.

The greedy algorithm is one of the simplest approaches to solve the optimization problem in which we want to determine the global optimum of a given function by a sequence of steps where at each stage we can make a choice among a class of possible decisions. In the greedy method the choice of the optimal decision is made on the information at hand without worrying about the effect these decisions may have in the future. Greedy algorithms are easy to invent, easy to implement and most of the time quite efficient [14].

\subsection{Standard Greedy Method/Quadratic Greedy method}

The standard greedy algorithm $G$ for solving the SSP starts with an empty solution-subset and examines the numbers wi in the non-increasing order of their values. Each considered wi is inserted into the current solution if and only if it is smaller than the difference between $\mathrm{K}$ and the sum of the current solution. The time complexity of the greedy algorithm is $\mathrm{O}(\mathrm{n} \log \mathrm{n})$ since it is dominated by the sorting operation, and the space required is $\mathrm{O}(\mathrm{n})$. The worst-case performance ratio is equal to $1 / 2$.

Martello \& Toth [10][15] presented another greedy method with better worst- case performance: the quadratic greedy (QG) algorithm. The idea is to apply the greedy algorithm $\mathrm{n}$ times, by considering the sorted item sets $\{1 \ldots, \mathrm{n}\},\{2, \ldots, \mathrm{n}\}$, $\{3 \ldots, n\}$, and so on, respectively, and take the best solution . The running time of QG is $\mathrm{O}(\mathrm{n} 2)$, its space complexity is $\mathrm{O}(\mathrm{n})$ and the worst-case performance ratio is equal to $3 / 4$.

More formally let's consider XG(i) and zG(i) respectively corresponding to the optimal solution (the set of items to be selected) and the achieved profit obtained at the ith iteration. The simple greedy algorithm G(i) applied to the ith iteration is as follows:

\section{Procedure $\mathbf{G}^{(\mathbf{i})}$}

Input $\mathrm{N}, \mathrm{K}, \mathrm{w}_{\mathrm{j}}, \mathrm{i}$

Output $z^{G(i)}, X^{G(i)}$

1: Initialization: $\mathrm{X}^{\mathrm{G}(\mathrm{i})}=\emptyset, \mathrm{K}^{\prime}=\mathrm{K}, \mathrm{z}^{\mathrm{G}(\mathrm{i})}=0$.

2: for $\mathrm{j}=\mathrm{i} . . \mathrm{n}$ do

3: $\quad$ if $w j<K^{\prime}$

4: $\quad \mathrm{X}^{\mathrm{G}(\mathrm{i})}=\mathrm{X}^{\mathrm{G}(\mathrm{i})} \mathrm{U}\{\mathrm{j}\}$

5: $\quad \mathrm{K}^{\prime}=\mathrm{K}^{\prime}-\mathrm{w}_{\mathrm{j}}$

6: $\quad$ end if

7: $\quad \mathrm{z}^{\mathrm{G}(\mathrm{i})}=\mathrm{K}-\mathrm{K}^{\prime}$

8: end for

Then the quadratic greedy algorithm QG is formulated as a simple iteration of G(i) :

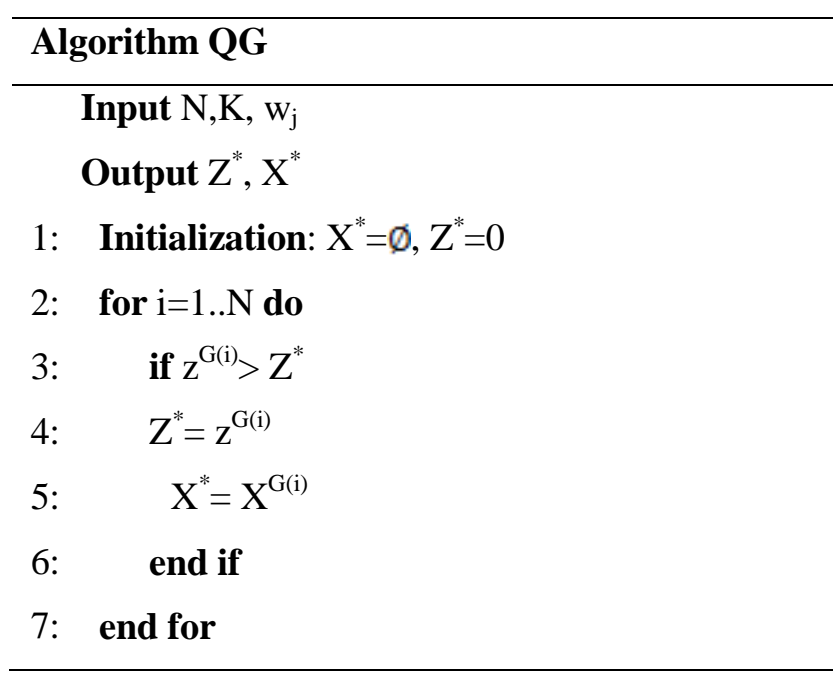

Where $\mathrm{X}^{*}$ the optimal channel set to be selected and $\mathrm{Z}^{*}$ is the total transmitted amount of data during $\min \left\{\mathrm{x}_{\mathrm{i}} \mathrm{HT}^{(\mathrm{i})}\right\}_{\mathrm{i}=1 . . \mathrm{N}}$.

Besides to applying the original notation of our problem formulation, some modification are proposed to the standard greedy algorithm in order to fit the specificity of our problem namely:

- the cardinality constraint through adding a condition about the number of channels selected: $c h$.

- $\quad$ and the inter-item dependency through updating at each iteration the value of $\mathrm{w}_{\mathrm{j}}$.

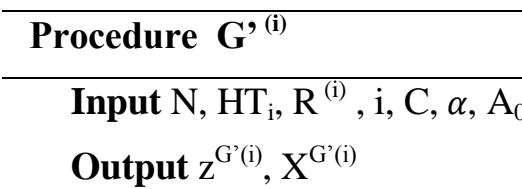

1: Initialization: $\mathrm{X}^{\mathrm{G}^{\prime}(\mathrm{i})}=\emptyset, \mathrm{K}^{\prime}=\mathrm{K}, \mathrm{z}^{\mathrm{G}^{\prime}(\mathrm{i})}=0, \mathrm{xj}=1$ for all $\mathrm{j}=\mathrm{i} . \mathrm{N}, \mathrm{ch}=0 / /$ number of channels selected

2: $\quad$ for $\mathrm{j}=\mathrm{i} . . \mathrm{N}-\mathrm{C}+1$

3:

$$
\mathrm{w}_{\mathrm{j}}=\sum_{\mathrm{k}=\mathrm{i}}^{\mathrm{j}} \mathrm{x}_{\mathrm{k}} \mathrm{R}^{(\mathrm{k})} \times \min \left\{x_{i} H T^{(i)}\right\}_{\substack{i=1 . N \\ x_{i}=1}}
$$

4: $\quad$ if $\mathrm{w}_{\mathrm{j}} \leq \alpha \mathrm{A}_{0}$ and $\mathrm{ch}<\mathrm{C}$

5: $\quad \mathrm{X}^{\mathrm{G}^{\prime}(\mathrm{i})}=\mathrm{X}^{\mathrm{G}^{\prime}(\mathrm{i})} \mathrm{U}\{\mathrm{j}\}$

6: $\quad \mathrm{ch}=\mathrm{ch}+1$

7: $\quad$ else $x j=0$

8: $\quad$ end if

9: $\quad$ if $\mathrm{ch}=\mathrm{C}$

10:

$$
\mathrm{z}^{\mathrm{G}^{\prime}(\mathrm{i})=} \mathrm{W}_{\mathrm{j}}
$$

11: end if

12: $\quad$ end for 
The same iteration procedure is applied to G'(i) to get the quadratic greedy algorithm results.

It's worth mentioning that the greedy search has to be applied to a sorted list of items. For the sorting operation, Martello and Toth proposed to sort items according to item values wj however in our case such sorting is not possible because of the interdependency between $\mathrm{wj}$, then three alternatives are proposed:

- To sort the channels (the items) in an optimistic manner without regard to dependencies. Channels are thus sorted individually on the basis of the value $H T^{(j)} \times R^{(j)}$.

- To sort the channels according to the dependencies in the value function such that channel i always precedes channel $\mathrm{j}$ if its value depends on $\mathrm{j}$. Channels are thus sorted on the basis of the value $H T^{(j)}$.

- $\quad$ To sort the items according to $R^{(j)}$.

\subsection{Proposed multichannel access algorithm}

The channel selection strategy fulfilling the above mentioned access model:

- The CR estimates the (HT) corresponding to each channel through a sensing /learning phase or a common control channel implementation.

- Estimates the achievable rate corresponding to each channel when transmitting with the maximum allowed transmission power.

- Determines the optimal channels to transmit on through applying the quadratic greedy algorithm detailed above.

- Transmits over the set of channels $X^{*}$ until a PU appears and reclaims one of the selected channels.

At each $\mathrm{HO}$ the parameter $A_{0}$ is updated as follows:

$$
A_{0}=A_{0}-H T_{e f f} \times \sum_{i=1}^{N} x i * R^{(i)}{ }_{e f f}
$$

where $\mathrm{HT}_{\text {eff }}$ and $\mathrm{R}_{\text {eff }}$ are respectively the effectively perceived $\mathrm{HT}$ and rate.

\section{PERFORMANCE EVALUATION}

\subsection{Simulation Setup}

Numerical simulations are launched using Matlab, to test the effectiveness of the proposed access model in reducing the number of HOs needed to achieve SU transmission. In our simulations, we considered a matrix of binary following a poissonian arrival as a representative sample of spectrum usage. Each line of one matrix refers to a given channel and each column corresponds to a timeslot $\Delta$ t. The 0 state corresponds to a spectrum hole while the 1 corresponds to a busy state. We consider different arrival rates among the channels (to obtain different HT values), and to each channel we associate randomly a rate value in the interval $[100 \mathrm{kbps}$ $1 \mathrm{Mbps}]$.

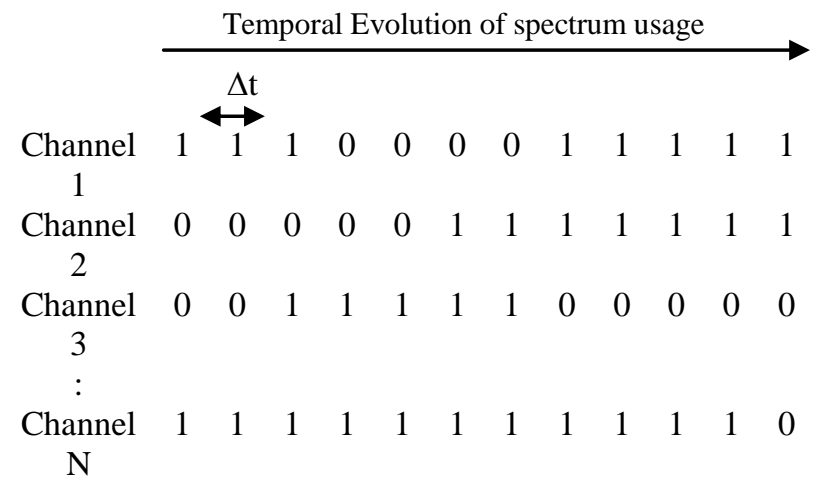

A SU is supposed accessing the spectrum composed of 30 non overlapping channels according to the proposed model and selecting $\mathrm{C}=5$ channels simultaneously. The $\mathrm{SU}$ has an amount of data $\mathrm{A}=500 \mathrm{Mb}$

\subsection{Simulations results}

To evaluate the performance of the proposed model, we compare first the three access strategy corresponding to the sorting criterion over which the greedy algorithm is based namely:

- $\quad$ HT-Rate access model (HTRAM) where the access is done according to the optimistic sorting strategy combining HT and Rate. This selection strategy favors selecting channels having highest achievable amount of transmitted data during HT that is : $H T_{j} \times R^{(j)}$.

- $\quad$ HT based model (HTAM) in which the sorting and the selection strategies favor selecting the channel having longer HT.(independently of the achievable rate).

- Achievable Rate Access model (ARAM) in which the SU tries to reduce the needed number of HOs by selecting channels offering the best achievable rates. The sorting strategy related to the greedy algorithm is based on the rate criterion.

These three sorting and access strategy were compared according to the optimality of the solution $\mathrm{Z}^{*}$ and the number of iterations needed to reach the optimal solution.

As depicted in figure (1), HTRAM optimal solution outperforms the HTAM solution of about $5 \%$ and exceeds noticeably the ARAM solution of about $42 \%$. 


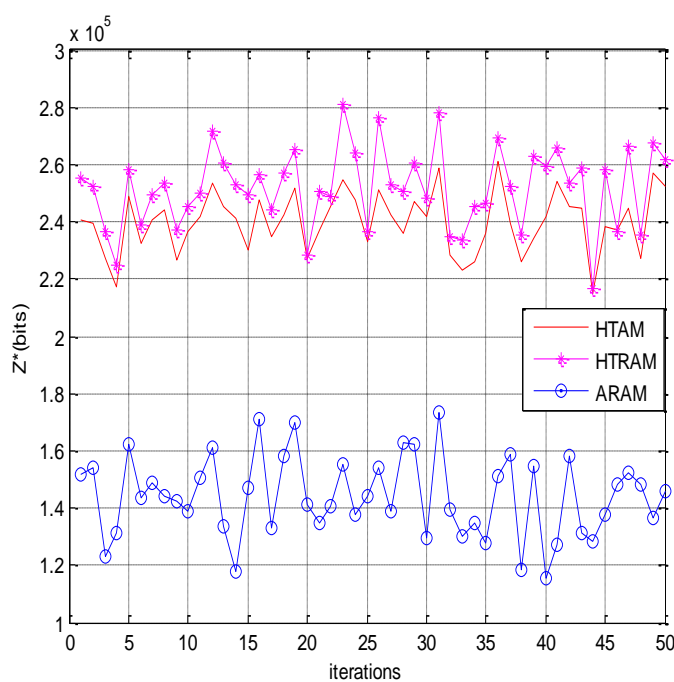

Figure 1: Comparison of the optimal solution $\mathrm{Z}^{*}$ obtained using the 3 access models

This performance has been tested for several dispersion values of HT and Rate. Measures of dispersion indicate how the values of a variable are spread out around the central value. That is to ensure that this optimality does not depend on the HT and Rate dispersion range.

In this work, a relative measure of dispersion is considered: the coefficient of dispersion corresponding to the ratio of standard deviation to the mean of a data set. We focused on this work on CV (HT) :coefficient of dispersion of HT values and CV(R) coefficient of dispersion of rate values.

It can be observed from figure (2) and figure (3) that in most scenarios, the HTRAM quadratic greedy algorithm yields the best performance independently with HT and rate dispersion.

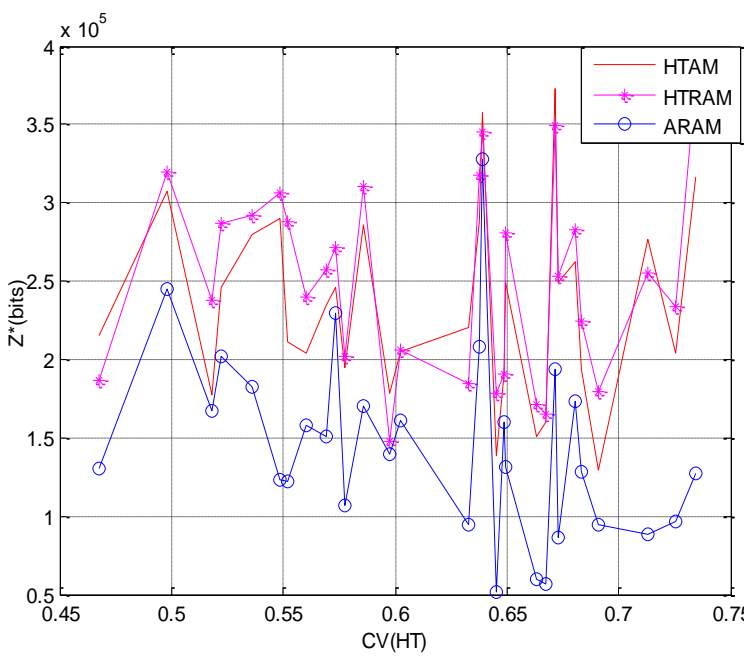

Figure 2: Optimality of the solution according to CV(HT)

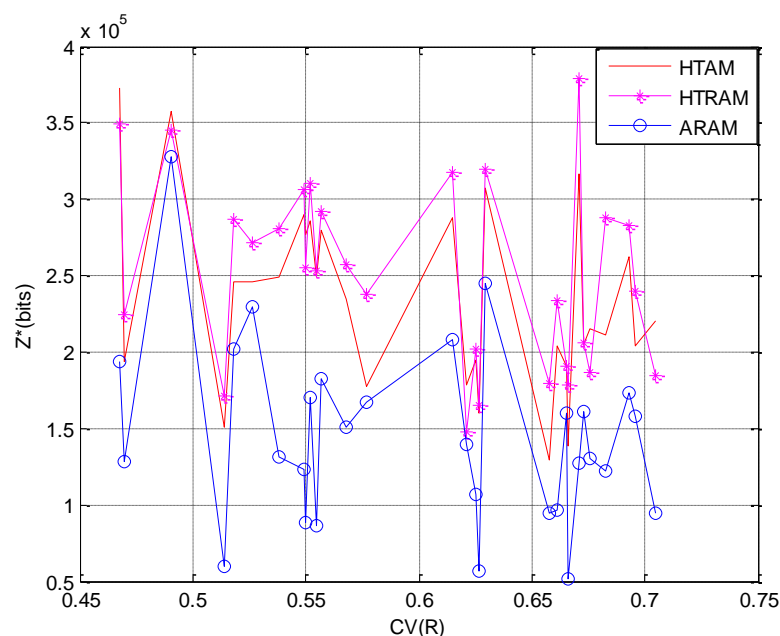

Figure 3: Optimality of the solution according to CV(R)

Now if we come back to the goal of our proposed access model which is reducing the needed number of HOs to achieve SU transmission.

Simulations show that the HT-Rate based access model (HTRAM) reduced the needed number of handoffs of about $26 \%$ compared to ARAM and 3\% compared to HTAM. It is important to mention that HTAM outperforms ARAM of about $23 \%$ in terms of reduction in needed HOs, which means that a HT maximizing access scheme is more efficient than a rate maximizing scheme specially in case of multichannel access.

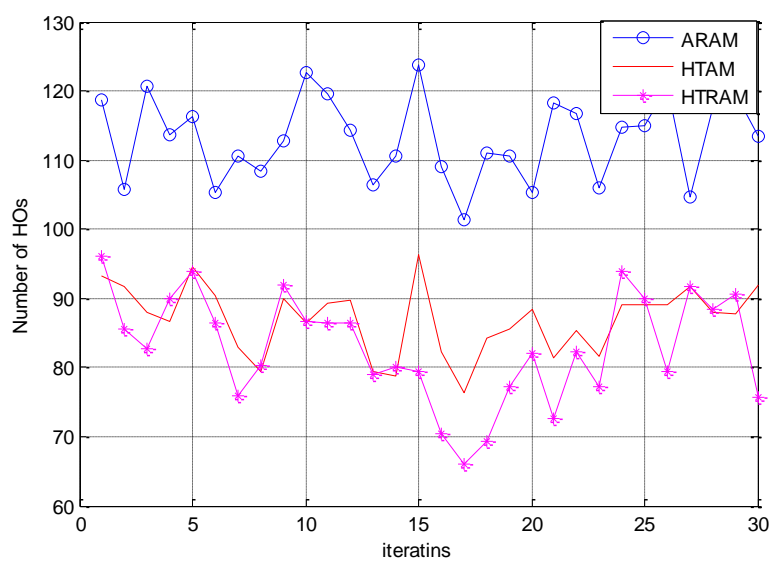

Figure 4: Effectiveness of HTRAM compared to other models (RAM, HTAM, ARAM) in reducing the number of HOs

On the other hand, we have checked the number of iterations needed to reach the optimal solution for all the 3 proposed access schemes. As depicted in figure (4), the HTRAM is the most rapid strategy since it needs from 1 to 2 iterations to get the optimal solution which mean that the standard greedy algorithm is applied only 1 to 2 times, thus no need to iterate $\mathrm{N}$ times, we could then stop on the 2 nd try at most. This reduces considerably the time complexity of the quadratic algorithm. 


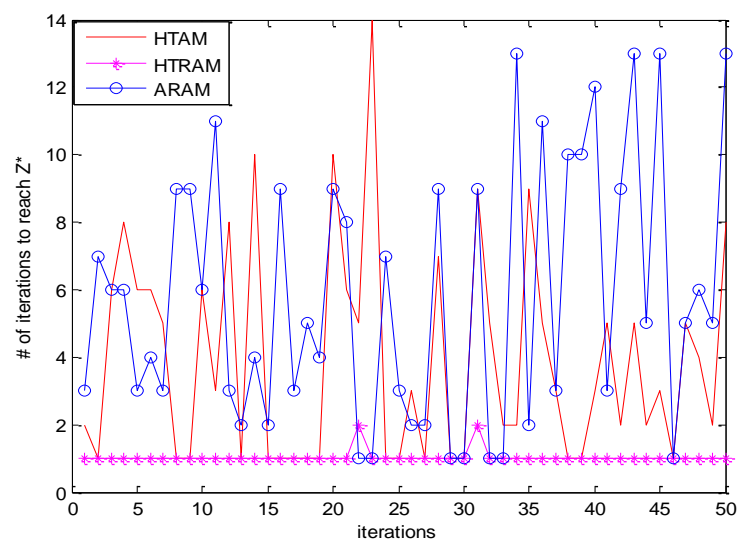

Figure 5: Comparison of number if iteration needed to reach the optimal solution

\section{CONCLUSION}

In this paper, the problem of minimizing the HO process due to PU activity in case of multichannel access is investigated. We modeled the problem as subset sum problem with cardinality constraint and inter-item dependency. We introduced a channels selection strategy fulfilling the optimization constraints and verified through numerical simulations the effectiveness of the proposed algorithm based on joint HT and rate consideration. This work dealt with the case of single SU, this allowed to validate the proposed model As future work, it will be interesting to extend the problem formulation to deal with the case of concurrent SU access to spectrum. In fact, favoring channels having jointly best HT and Rate may increase contention between several SUs. An interesting future issue is to solve such contention using a game theoretic framework allowing to establish an adaptation algorithm on the basis of which a Nash equilibrium is reached.

\section{REFERENCES}

[1] P. Li, N. Scalabrino, and Y. Fang, "Channel switching cost in multichannel wireless mesh networks," in Milcom'07.

[2] I. F. Akyildiz, w.-Y. Lee, M. C. Vuran, and S. Mohanty, "NeXt generation/dynamic spectrum access/cognitive radio wireless networks: A survey," Computer Networks (Elsevier), vol. 50, pp. 2127-2159, September 2006.

[3] D. Wilikomm, J. Gross, and A. Wolisz, "Reliable link maintenance in cognitive radio systems," in Proc. IEEE DySPAN, November 2005, pp.371-378.
[4] W. Hu, D. Willkomm, G. Vlantis, M. Gerla, and A. Wolisz, "Dynamic frequency hopping communities for ecient ieee 802.22 operation," IEEE Communications Magazine, pp. 80-87, May 2007.

[5] c.-w. Wang and L.-C. Wang, Modeling and analysis for reactive-decision spectrum handoff in cognitive radio networks," in Proc. IEEE GlobeCom, 2010.

[6] c.-w. Wang and L.-C. Wang, "Modeling and analysis for reactive-decision spectrum handoff in cognitive radio networks," in Proc. IEEE GlobeCom, 2010.

[7] L. Yang, L. Cao, and H. Zheng, "Proactive channel access in dynamic spectrum networks," PhYSical Communication (Elsevier), vol. I, pp.103-111, June 2008.

[8] Dan Xu, Eric Jung, and Xin Liu: 'Optimal Bandwidth Selection in Multi-Channel Cognitive Radio Networks: How Much is Too Much?' IEEE Symposium on New Frontiers in Dynamic Spectrum Access Networks (DySPAN) 2008, Chicago.

[9] H. Kellerer, U. Pferschy, and D. Pisinger, Knapsack Problems.Springer, 2004.

[10] S. Martello and P. Toth, Knapsack Problems: Algorithms and Computer Implementations. Wiley, 1990.

[11] Martello, Silvano, David Pisinger, and Paolo Toth. "New trends in exact algorithms for the $0-1$ knapsack problem." European Journal of Operational Research 123.2 (2000): 325-332.

[12] Burg, Jennifer J., et al. "Experiments with the "Oregon Trail Knapsack Problem"." Electronic Notes in Discrete Mathematics 1 (1999): 26-35.

[13] Caprara, Alberto, et al. "Approximation algorithms for knapsack problems with cardinality constraints." European Journal of Operational Research 123.2 (2000): 333-345.

[14] Gonzalez, Teofilo F., ed. Handbook of approximation algorithms and metaheuristics. CRC Press, 2007.

[15] Martello, Silvano, and Paolo Toth. "Worst-case analysis of greedy algorithms for the subset-sum problem." Mathematical programming 28.2 (1984): 198205 . 